\title{
Cost efficient internet of things based smart farm system for rural farmers: Leveraging design thinking approach
}

\author{
Segun Adebayo ${ }^{1^{*}}$, Ozichi Emuoyibofarhe ${ }^{2}$, Tolulope Awofolaju ${ }^{3}$ \\ ${ }^{1}$ Mechatronics Engineering Program, College of Agriculture, Engineering and Sciences, Bowen University, Iwo, Nigeria \\ ${ }^{2}$ Computer Science Program, College of Computing and Communication Studies, Bowen University, Iwo, Nigeria \\ ${ }^{3}$ Electrical and Electronics Engineering, College of Engineering and Technology, Osun State University, Osogbo, Nigeria
}

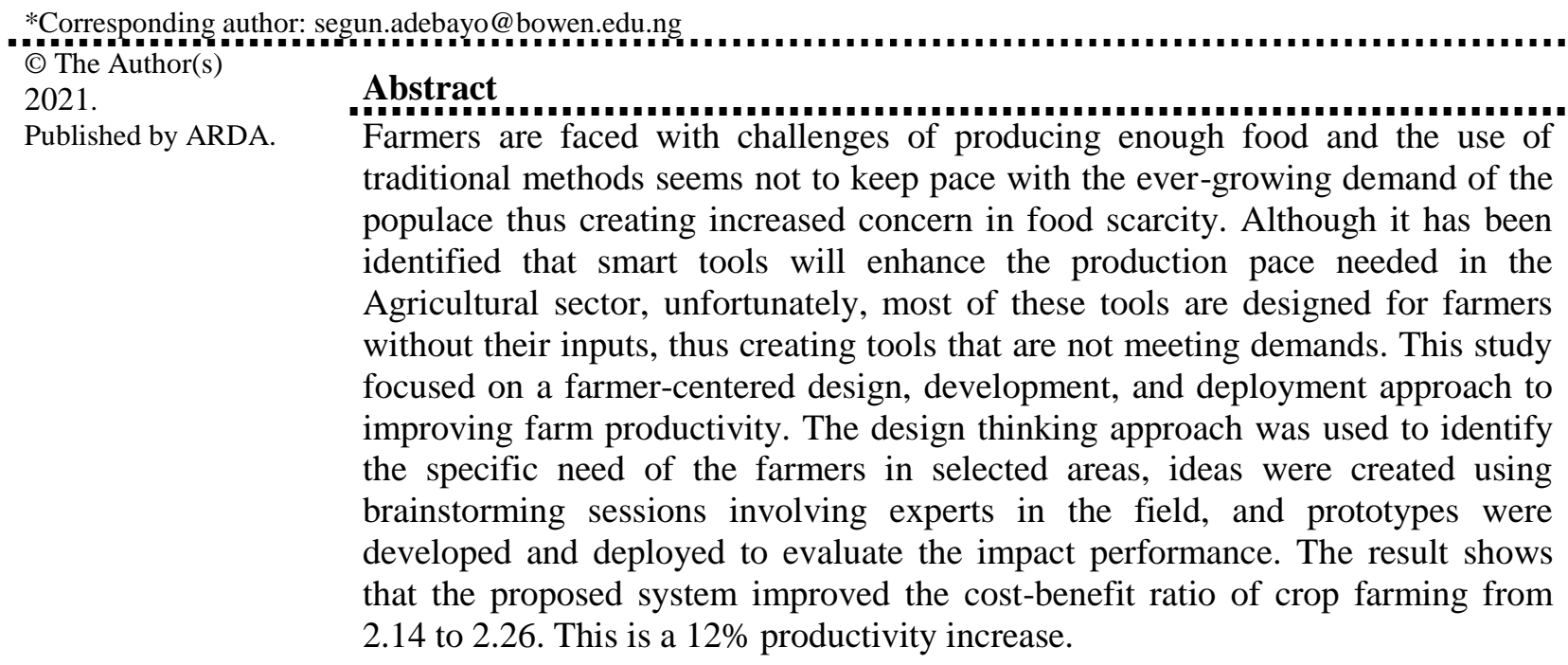

Keywords: Smart Farming, Sensor, Internet of Things, Design thinking, Unmanned Aerial Vehicle

\section{Introduction}

Agriculture is a major contributor of employment and well-being to the African population, especially the ones in the rural area. Study shows that agriculture contributes over $40 \%$ gross domestic product (GDP) and $60 \%$ employment to several countries. This sector is not without its challenges and most solutions developed to address these challenges are not effective because the development process is not user-centered.

Design thinking is a solution-based approach to problem-solving through human problems understanding. The problems identification is human centered, ideas geared toward solving these problems are suggested in a brainstorming session, and finally, prototypes developed are tested and evaluated. Reference [1] identifies the design thinking technique as a way of finding human problems and creating solutions through the use of tools and experts' mindsets. Reference [2] recognizes the approach as a creative, human-centered, collaborative, iterative, and visual way of proffering solutions to problems. Human intuitiveness, ability to recognize patterns and create ideas, are very useful in this approach.

\section{Methodology}

The study employed the use of design thinking to address the challenges peculiar to agriculture in rural areas. The process, as shown in Fig. 1, ranges from empathy, problem definition, idea formation, prototype development and product testing among the users for feedbacks in order to fine-tune the product's performance.

This work is licensed under a Creative Commons Attribution License (https://creativecommons.org/licenses/by/4.0/) that allows others to share and adapt the material for any purpose (even commercially), in any medium with an acknowledgement of the work's authorship and initial publication in this journal. 


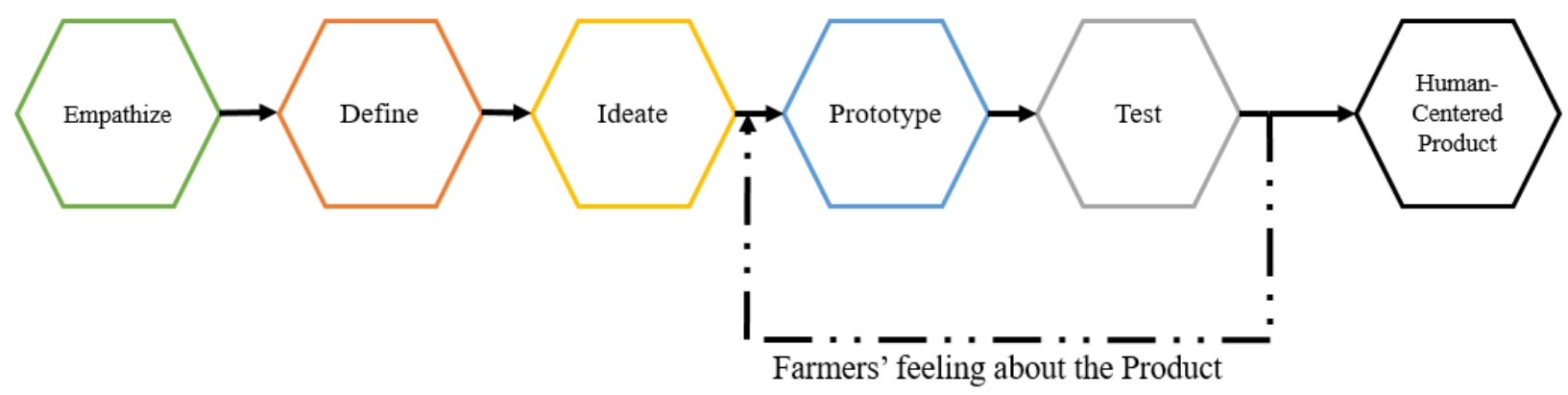

Figure 1. Design thinking-based solution development process

The study visited rural dwellers whose major occupation is farming in selected areas of Nigeria. These areas were chosen based on the categorization of Nigeria into sections as shown in Fig. 2.

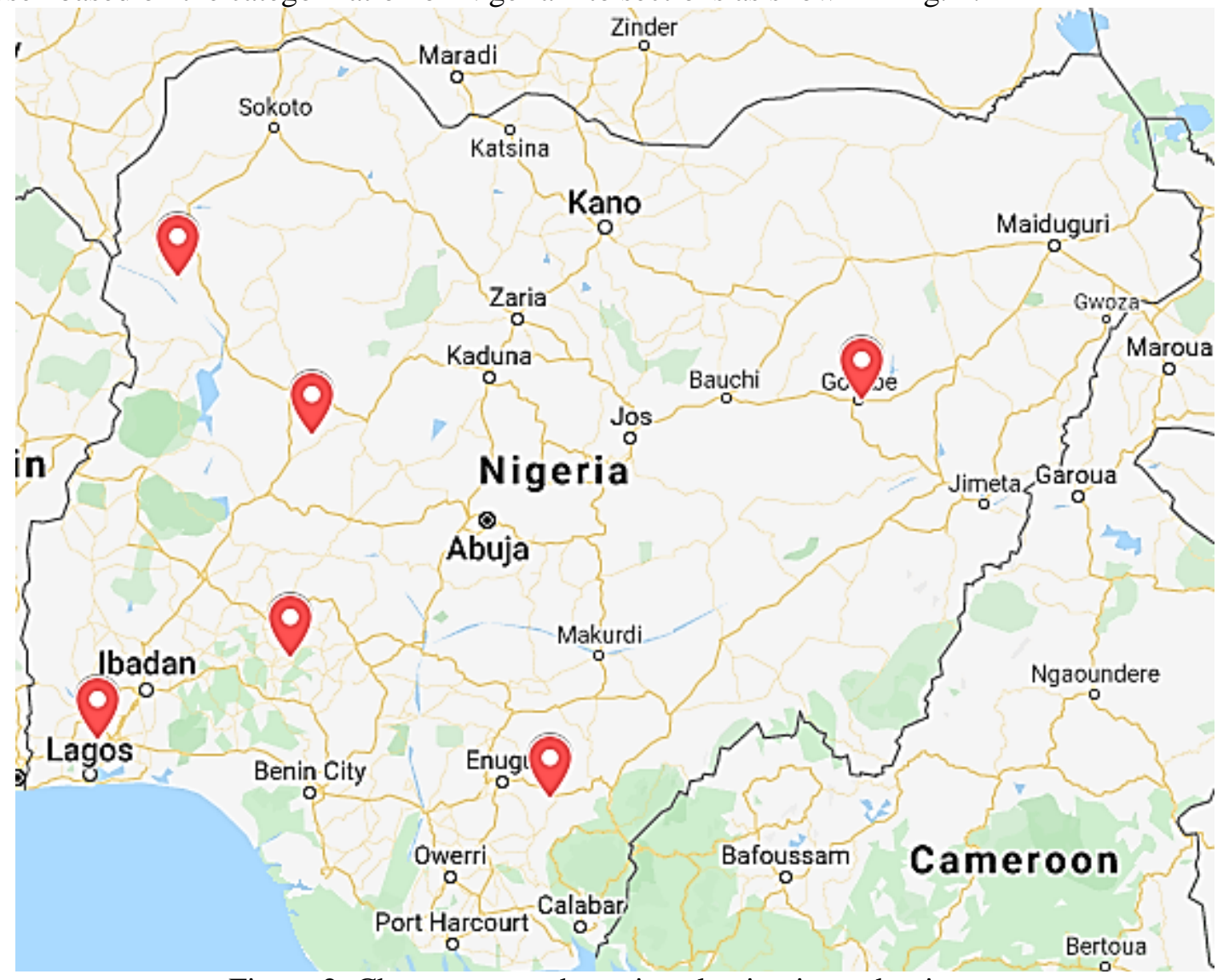

Figure 2: Chosen areas where rice planting is predominant

\subsection{Empathy}

This process creates the platform of interacting with the key players in the domain in order to listen and hear various needs and desires. It helps in the creation of innovative and inclusive solutions geared toward meeting the demand of the targeted audience. It helps in delivering an affordable, accessible, and sustainable solution. The study consulted two major groups at this stage: local farmers and agricultural extension experts, to find out problems encountered by farmers. This was done using both structured and semi-structured interviews as well as observations. The focus was to gain enough knowledge about farmers' experiences. Relevant information regarding farmers' challenges on farm field monitoring and management were captured.

\subsection{Problem definition}

This stage availed the opportunity of putting together the information acquired during the empathy stage. Information were synthesized and analyzed in order to narrow down the main problems to be addressed. The study was able to categorize the problems facing famers into three major groups as shown in Fig. 3 . Information showed that local farmers are faced with several challenges ranges from insufficient capital to 
grow large hectares of lands, low-yield farming system techniques, biotic/abiotic stress conditions, and insufficient improved crop varieties [3]. The above findings lend support to the study by [4] which revealed that several factors, such as input, un-mechanized production process, pest infestation, and so on, constitute the challenges that affects farming.

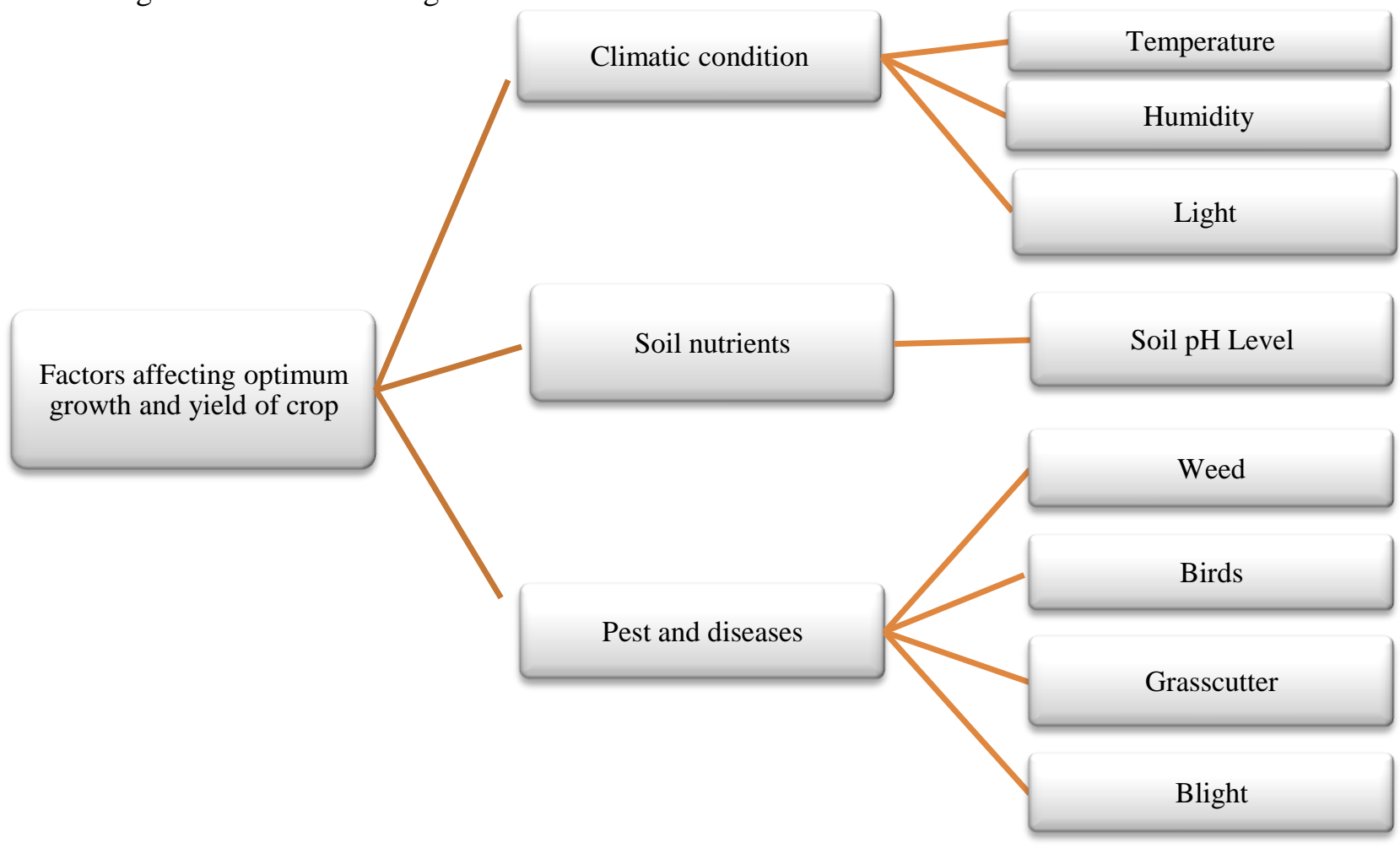

Figure 3. Categorization of factors affecting farming system

\subsection{Idea creation}

This stage involved generating ideas using defined problems. Team members discussed interesting design ideas that could enhance farmers' management capability through brainstorming sessions. The use of paper sketches, scenarios and mini-prototypes were helpful in illustrating and visualizing ideas. A system architecture as depicted in Fig. 4 proposes sensor placement on farm fields to determine and record environmental changes. These changes are then sent to a base station for both local and cloud storage as well as actuator response. The base station activates autonomous agents to address the changes at the right time and in the right location using the right amount of resources. Social media app such as Twitter, Facebook or WhatsApp can be connected to the cloud service for real time updates. The complete architecture can be categorized into five layers: physical, communication, computing, storage, service management, and application layer. The physical layer consists of the wireless sensor node deployed on the farm, the actuators (drone), and the controllers (base station). In this layer, studies [11] have focused on increasing computational processing capabilities, and reducing electronic component power consumption. The communication layer dealt with the communication among the sensor nodes known as device to device (D2D) communication and communication between a sensor node and the BS. The reliability, dexterity and effective performance of the whole architecture depends on this layer. The computing layer deals with the data processing for decision making at the BS before it get to the cloud or the remote location. This layer provides timely reaction of the system to prevent loss. The storage layer deals with information obtained from BS at the computing layer which stored. There is the need for adequate protection of these data terms of security and integrity. This layer also handles other sub layers such as network management, cloud computing, and data analytics. The application layer is the layer visible to the user. The software interacts with other layers to give the farmer up to date and timely information about the farm for proactive action that will yield good result. 


\section{Prototype development and experimental setup}

The ideas presented in the previous sections were implemented as prototypes for proper investigation, evaluation, and improvement. and re-examined, or rejected on the basis of the users' experiences. The aim is to have a better understanding of the constraints inherent to the solution and the problems at hand. For simplicity, the solution was deployed on a rice farm. Rice crop has been considered as one of the most consumed food crop of the developing countries as well as more than half of the world's population [12].

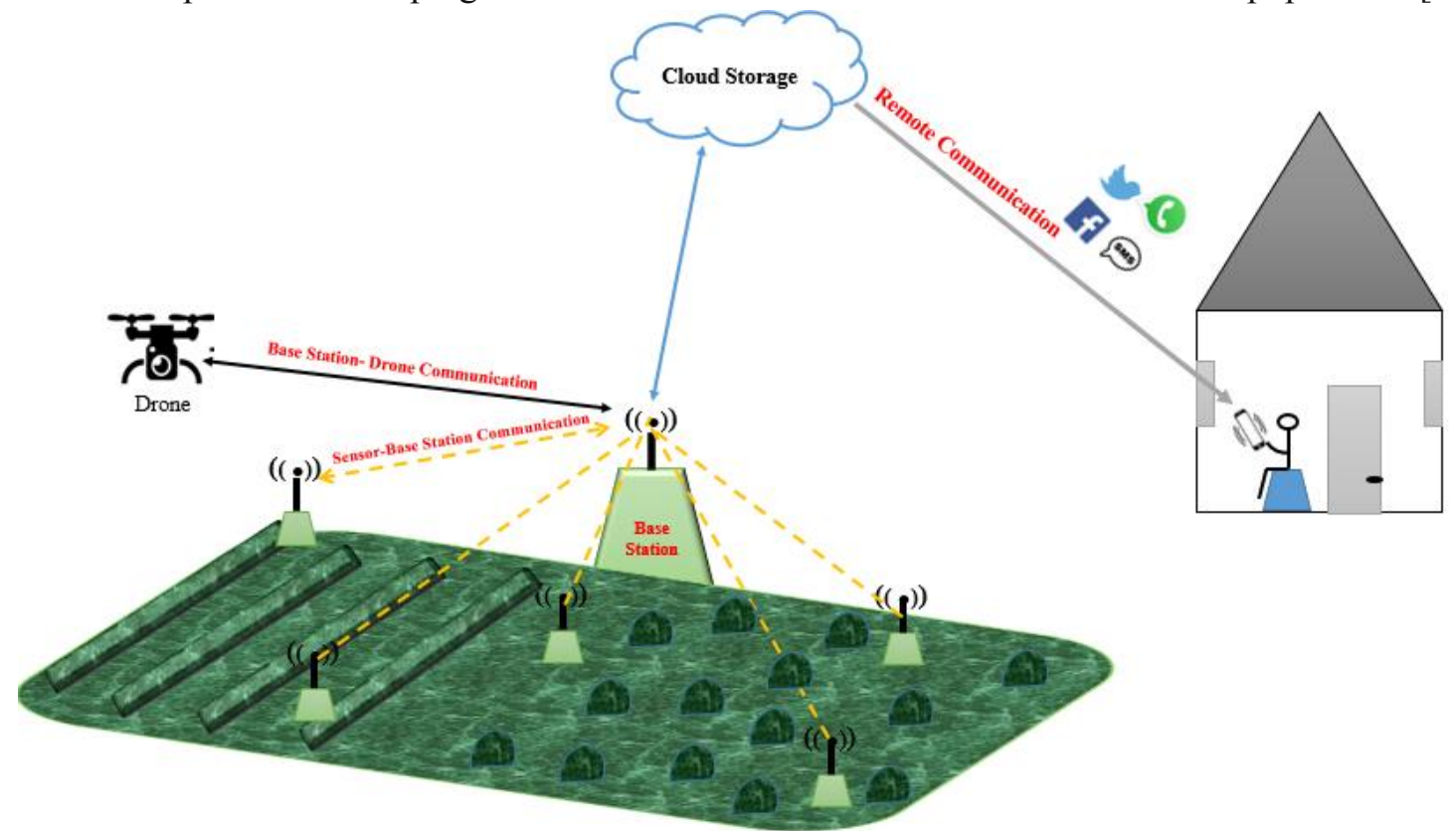

Figure 4. Smart farm system architecture

\subsection{Sensor node}

Sensor nodes are the devices placed in the environment to monitor changes in the environment. The hardware components of a sensor node include: sensing, processing, transmitting, and power units. The sensing unit consists of both the sensors such temperature, moisture, etc. and an analog-to-digital converter (ADC). ADC converts analog signals generated by the sensors to digital signal for processing. A sensor node can have a combination of sensors for a smarter device. The processing unit performs computation on the signals received from the sensing unit. These computations may include comparison, storage or transmission. The transmitting unit connect the node to the network and helps in sending signals to and fro. The power unit supply energy needed by the electronic components of the node. The major source of energy for sensor nodes are batteries, although recently, solar energy and other forms of renewable energy had been introduced [5, 13, 14]. It has been noted that sensor node should be designed such that it consumes minimum energy in order to extend the life time.

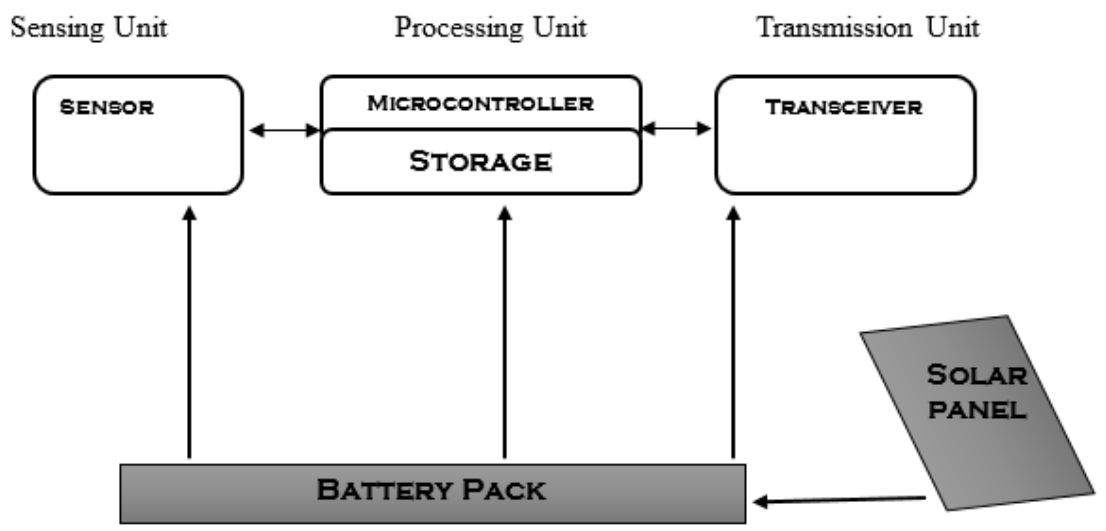

Figure 5. Typical components of a sensor node 
In an attempt to design an energy efficient sensor node, the hardware components were chosen carefully to obtain high efficiency with minimum hardware and cost. The sensors were chosen based on the identified parameters responsible for the yield of grain crops: atmospheric temperature and relative humidity, soil moisture and $\mathrm{pH}$, and pest sensors. Arduino Nano, a microcontroller board was used as the processing component. The board is based on Advanced Virtual Reduced Instruction Set Computer (AVR) chipset [15] and has a $32 \mathrm{~KB}$ internal built-in memory for storage. Arduino microcontroller can be programmed using the integrated development environment (IDE). A sensor node can be in transmitting, receiving, sleep or idle mode. Study [16] has shown that about $40.25 \%$ and $29.1 \%$ energy depletion occur when nodes are in transmitting and receiving modes respectively, while $25.3 \%$ and $5.4 \%$ energy is depleted when node is in idle and sleep modes respectively. Thus, an energy aware protocol will greatly enhance the network lifetime of any sensor network. Fig. 6 shows the energy aware protocol for the sensor interaction with the network.

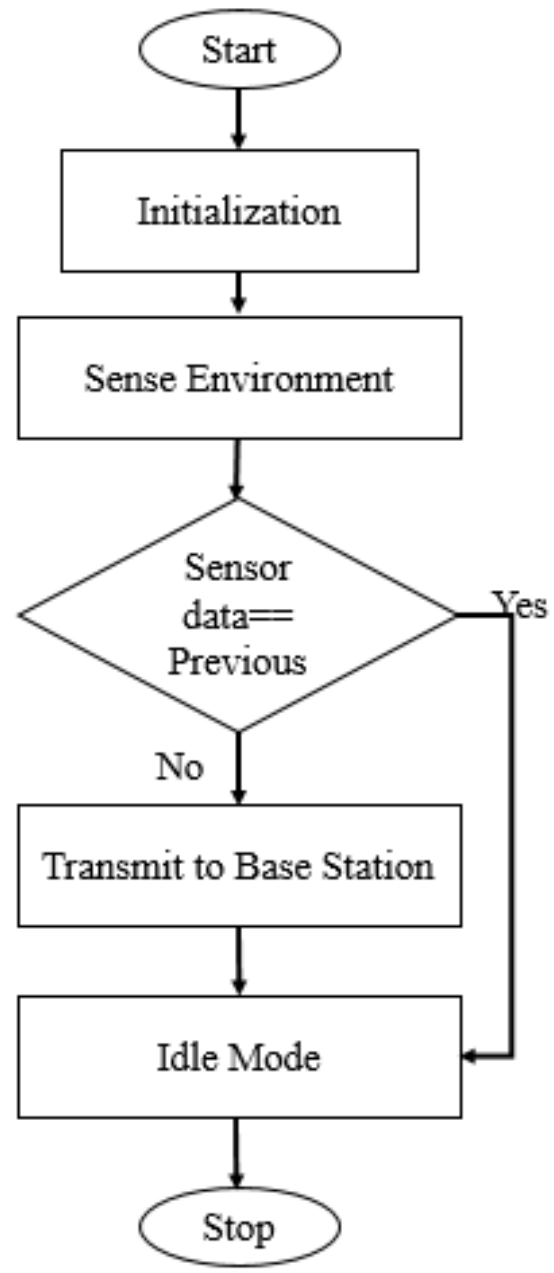

Figure 6. Flowchart of sensor node interaction protocol

\subsection{Sensor node placement}

Wrong placement of low energy consuming sensor nodes on farm field can defeat the promising features of wireless sensor network such as improved productivity, reduced cost, and resource optimization. A major cause of wrong sensor placement can be attributed to cost and range of each sensor [6]. Thus optimum placement of sensors in a field is a key factor to reaping the dividend of precision farming. In this study we examine Territorial Predator Scent Marking Algorithm (TPSMA) as proposed by [7]. TPSMA, a bio-inspired technique, operates on the principle of area marking by the predator based on factors such as food resources. Sensor node location can also be marked based on design objectives which may include coverage, energy consumption, and connectivity [7]. 
Let the monitored locations $i=1,2,3, \ldots, S$ and the potential sensor node location be given as $j=$ $1,2,3, \ldots, P$. The objective function $f(x)$ for wireless sensors network is the coverage ratio and it is given as

$$
f(x)=\frac{N_{m}}{N_{\text {all }}}
$$

where $N_{m}$ is the number of locations that are monitored and $N_{\text {all }}$ is the total number of locations. The algorithm to place sensor on cultivated farm is described in Table 2.

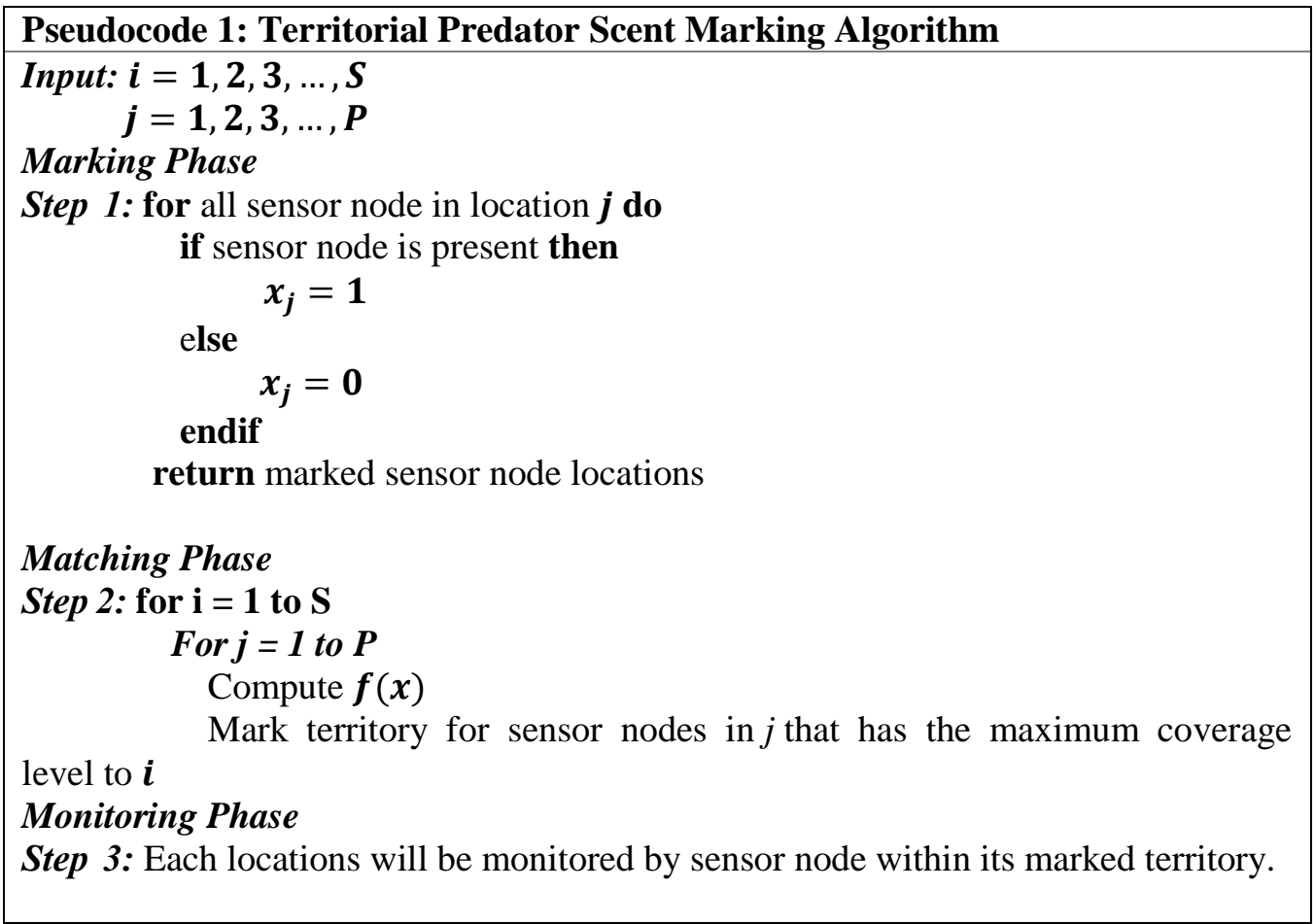

\subsection{Base station}

The base station is a low energy consuming computer board called Raspberry Pi of model B. The board is a micro-computer with several interfacing peripherals such as universal serial bus (USB) port, high definition multimedia interface (HDMI) port, 8-bit general-purpose input/output (GPIO) port for expansion and so on. Python scripts were developed to run on the system in order to receive data from field, store same in a comma separated value (csv) file and also post the data to a cloud storage using an application programming interface (API) key and feed id. The BS was setup such that data are logged every 30 minutes. This gives an average of 48 logs per sensor node in a day.

\subsection{Cloud server}

A number of cloud storage providers for internet of things (IoT) applications are emerging and these have appeared in several literatures [8-10]. Selecting the right cloud server for specific application depend on factors such as complexity, availability, finance and so on. ThingSpeak server was chosen as the cloud storage server due to it features [17]. The platform allows user to aggregate, visualize and analyze live data in the cloud and can be connect to mathematical analytic tool such as MATLAB. User can initiate instant visualization of live data alert on social media services such as twitter and so on.

\subsection{Farmers mobile application}

The implementation of the mobile application was done using android studios. Android was chosen as a platform for the implementation due to its availability and number of users. The android studio is powered majorly by java for the logic part and extended markup language (XML) for the frontend. Different libraries were integrated with the android studios which include android volley for making hyper-text transfer protocol 
(http) calls. In other for the system to function effectively, the mobile application makes the necessary API calls to the data source in the cloud. The cloud has a restful service which has been designed to handle different form of http calls including POST and GET. A simple algorithm resides on the mobile application which makes a judgment based on the data received. Analysis are performed on the data received and the farmer is advised based on the output of the algorithm. The frontend of the mobile application is the part of the mobile application which the user relates with.

\section{Experimental results}

\subsection{Wireless sensor network for precision farming}

Wireless sensors were deployed on a rice farm to measure environmental changes such as atmospheric temperature and humidity, and soil temperature, moisture and pH. Fig. 7 shows the implemented hardware and software architecture. The environmental values are sent, using wireless channel, to the base station. The base station logs data to the cloud using API key. Simple mobile application running on farmer's mobile phone access this information on the cloud to provide relevant suggestions to the farmer on irrigation, fertilization and crop rotation scheduling.

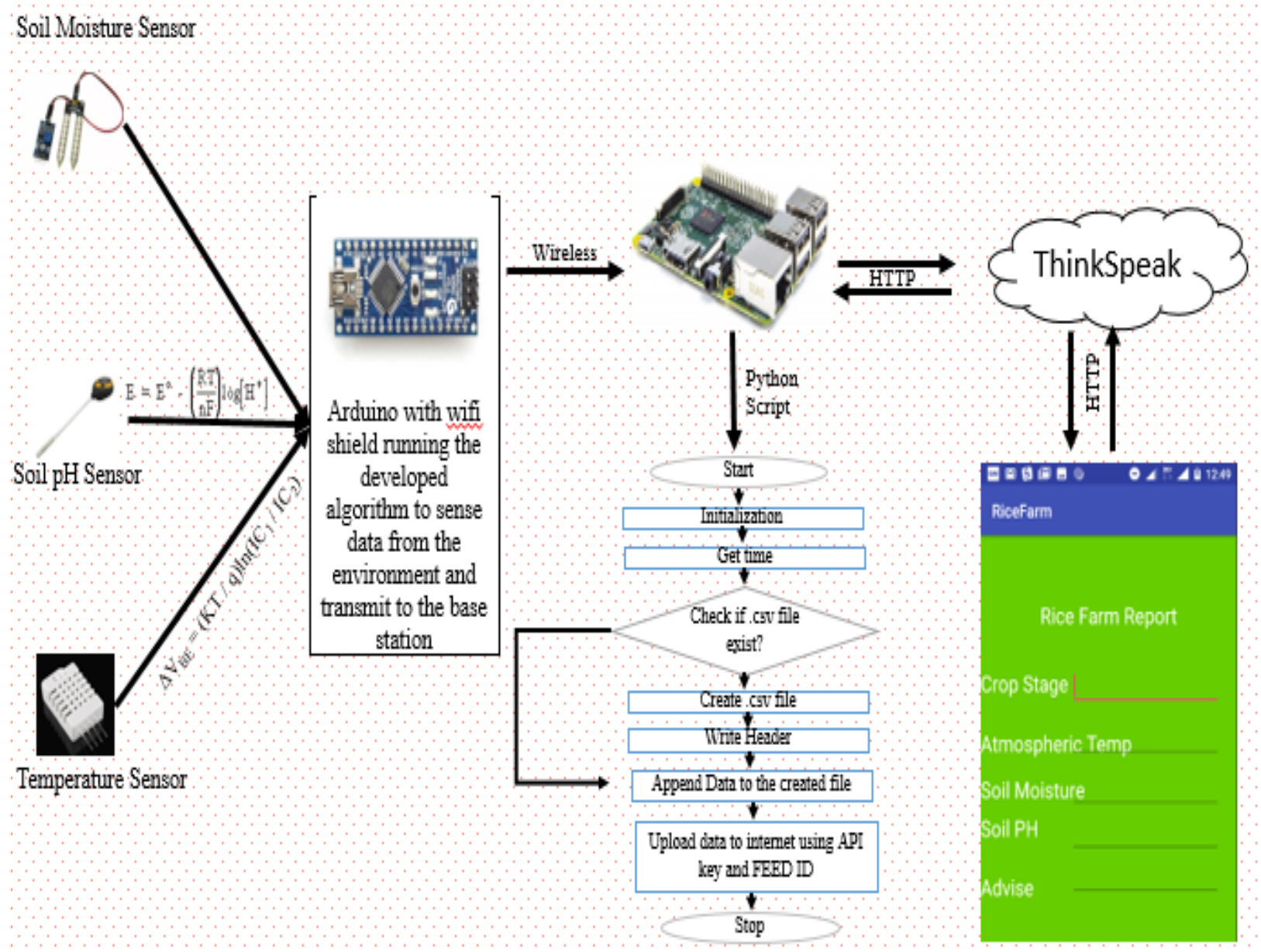

Figure 7. Smart farm hardware and software setup 


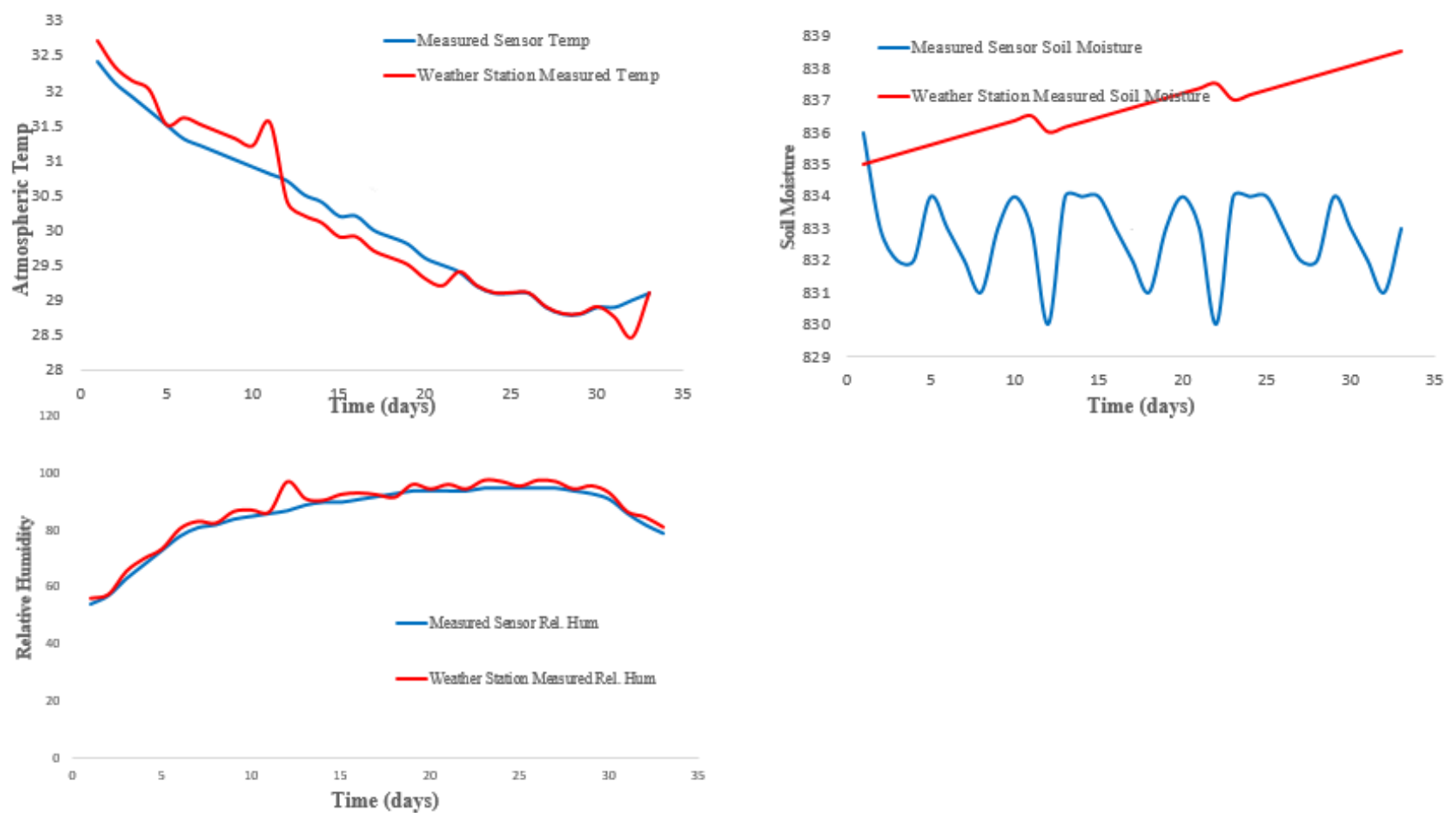

Figure 8. Sample of logged data

\subsection{Evaluation}

The effect of developed product deployment was evaluated using benefit and cost ratio (BCR). The study considered the benefit value of cultivating rice on a one-hectare land using both the conventional method and the proposed smart farm system. Table 1 shows the study survey that BCR using conventional method amounted to 2.14. This implies that for every unit cost, the farmer will get a benefit of 2.14. The result also shows that using proposed smart farm system a return of 2.32 is obtained for every unit cost. This shows an increase of $12 \%$ over the conventional method. Furthermore, it can also be deduced that there are benefits gained on reduction in wastage in applying farm inputs such as fertilizer and water. The reduction in wastage is attributed to real time information about the farm field status which will eventually translate to an increase in crop yield due to proper monitoring of the crop growth. Finally, although farming is profitable, more profit can be earnest using the proposed smart farm system.

Table 4: Cost rice production with and without precision farming (PF)

\begin{tabular}{|c|c|c|c|c|}
\hline Description & Quantity & $\begin{array}{l}\text { Unit cost } \\
\text { (\#) }\end{array}$ & $\begin{array}{l}\text { Total Cost } \\
\text { without PF ( })\end{array}$ & $\begin{array}{l}\text { Total Cost } \\
\text { with PF ( })\end{array}$ \\
\hline \multicolumn{5}{|l|}{ REVENUE: } \\
\hline Paddy rice yield (output) & $2,026.5 \mathrm{~kg}$ & 200 & 405,300 & 405,300 \\
\hline Total Benefit (A) & & & 405,300 & 405,300 \\
\hline \multicolumn{5}{|l|}{ VARIABLE COST (INPUTS): } \\
\hline Rice seeds & & 125 & 25,200 & 25,200 \\
\hline Fertilizer & 4 Bags & 7,000 & 28,000 & 28,000 \\
\hline $\begin{array}{l}\text { Agro-chemicals (pre and post } \\
\text { emergence }\end{array}$ & 10 Liters & 1,400 & 14,000 & 14,000 \\
\hline $\begin{array}{l}\text { Proposed smart farm system for } \\
\text { annum }\end{array}$ & & & 0 & 15,000 \\
\hline Ploughing, harrowing and leveling & 3 Man day & 5,000 & 15,000 & 15,000 \\
\hline Planting & 2 Man day & 1,500 & 3,000 & 3,000 \\
\hline Fertilizer application & 2 Man day & 1,000 & 2,000 & 2,000 \\
\hline Weeding herbicide application & 2 Man day & 1,500 & 3,000 & 3,000 \\
\hline Crop Protection & 30Man day & 1,500 & 45,000 & 0 \\
\hline
\end{tabular}




\begin{tabular}{|c|c|c|c|c|}
\hline Harvesting/threshing & 4 Man day & 5,000 & 20,000 & 20,000 \\
\hline Total Variable Cost (B) & & & 155,200 & 125,200 \\
\hline \multicolumn{5}{|c|}{ FIXED COST (DEPRECIATION): } \\
\hline Rent on Land & & & 11,900 & 11,900 \\
\hline Interest on loan & & & 14,600 & 14,600 \\
\hline Depreciation on machines used & & & 22,800 & 27,800 \\
\hline Total Depreciation (C) & & & 49,300 & 54,300 \\
\hline Total cost, $\mathrm{D}=\mathrm{B}+\mathrm{C}$ & & & 189,500 & 179,500 \\
\hline Gross margin, $\mathrm{E}=\mathrm{A}-\mathrm{B}$ & & & 250,100 & 300,100 \\
\hline Net returns, $F=E-C$ & & & 200,800 & 245,800 \\
\hline Benefit/Cost Ratio (A / D) & & & 2.14 & 2.26 \\
\hline
\end{tabular}

Source: Field Survey, $2018($ (\$1 = $\$ 363)$

\section{Conclusion}

The study had been able to successfully employ design thinking approach to understanding the needs of rural 8 farmers. The approach had been able to create a human-centered technological driven solution. Testing the 9 proposed solution had shown an increase in cost benefit ratio from 2.14 when using traditional method to 10 2.26 , using the proposed system.

\section{References}

[1] D. Kelley and T. Kelley, Creative Confidence: Unleashing the Creative Potential Within Us All, New York: Harper Collins Publishers, 2013.

[2] J. Maeda, "Design in Tech," Design in Tech Report, 2018.

[3] G. Waloszek, "Introduction to Design Thinking," 2012.

[4] E. Osabuohien, U. Okorie and R. and Osabohien, "Rice Production and Processing in Ogun State, Nigeria: Qualitative Insights from Farmers' Association," in Food Systems Sustainability and Environmental Policies in Modern Economics, IGI Gobal, 2018.

[5] R. Ibrahim, T. Chung, H. SM., B. K. and S. SK, "Solar energy harvester for industrial wireless sensor nodes," Procedia Computer Science, pp. 111-118, 2017.

[6] K. Visalini, B. Subathra, S. Srinivasan, G. Palmieri, K. Bekiroglu and S. Thiyaku, "Sensor Placement Algorithm With Range Constraints for Precision Agriculture," IEEE Aerospace and Electronic Systems Magazine, pp. 4-15, 2019.

[7] H. Zainol Abidin and N. M. Din, "Sensor node placement in wireless sensor network based on territorial predator scent marking algorithm," International Scholarly Research Notices, 2013.

[8] P. P. Ray, "A survey of IoT cloud platforms," Future Computing and Informatics Journal, pp. 35-46, 2016.

[9] T. Pflanzner and A. Kertész, "A survey of IoT cloud providers," in 39th International Convention on Information and Communication Technology, Electronics and Microelectronics (MIPRO), 2016.

[10] P. Ganguly, "Selecting the right IoT cloud platform," in 2016 International Conference on Internet of Things and Applications (IOTA), 2016.

[11] H. Rahimi, A. Zibaeenejad and A. A. Safavi, "A novel IoT architecture based on 5G-IoT and next generation technologies," in 9th Annual Information Technology, Electronics and Mobile Communication Conference (IEMCON, 2018.

[12] P. A. Seck, A. Diagne, S. Mohanty and M. C. Wopereis, "Crops that feed the world 7: Rice," Food security, pp. 7-24, 2012.

[13] M. Mabon, r. M. Gautie, B. Vrigneau, M. Le Gentil and O. Berder, "The smaller the better: Designing solar energy harvesting sensor nodes for long-range monitoring," Wireless Communications and Mobile Computing, 2019.

[14] S. Antony, S. Indu and R. Pandey, "An efficient solar energy harvesting system for wireless sensor network nodes," Journal of Information and Optimization Sciences, pp. 39-50, 2020. 
[15] S. Barrett and D. Pack, "Microchip AVR® Microcontroller Primer: Programming and Interfacing," Synthesis Lectures on Digital Circuits and Systems, pp. 1-383, 2019.

[16] M. Srbinovska, V. Dimcev and C. Gavrovski, "Energy consumption estimation of wireless sensor networks in greenhouse crop production," in IEEE EUROCON 2017-17th international conference on smart technologies , 2017.

[17] M. Maureira, D. Oldenhof and L. Teernstra, ThingSpeak-an API and Web Service for the Internet of Things, World Wide Web, 2011. 